\title{
XVII.
}

\section{Statistischer Beitrag zur operativen Eröffunng des Proc. mastoideus, resp. zur Radicaloperation bei Otitis media purulenta chronica.}

\author{
Vortrag, gehalten am 9. August 1899 auf dem 6. internationalen \\ otologischen (iongress in London. ${ }^{1}$ ) \\ Von
}

\section{A. Lacae,}

M. H. Als Einleitung zu meinem Vortrage kann ieh nioht genug hervorheben, dass ich auf die "Mastoidoperation" als ein ausserordentliches Heilmittel bei ehronischen Ohreiterungen einen hohen Werth lege, zumal ioh bei einer grossen Anzahl von Kranken nur mit Hülfe dieser Operation eine gründliche Heilung habe constatiren können. Die folgenden Beobachtungen sollen nur dazu dienen, den Missbrauoh der Operation möglichst einzuschränken.

In der unter meiner Direction stehenden Kg:l. UniversitätsOhrenklinik in Berlin sind vom April 1881 (Gründung der Anstalt) bis August 1899 im ganzen 1935 operative Eröffnungen des Proc. mastoideus vorgenommen worden, wovon 852 auf die acute und 1083 auf die chronische Form der Eiterung entfallen. Bei oberflächlicher Betrachtung dieser Zahlen könnten dieselben, selbst was die chronischen Fälle betrifft, etwas gross erscheinen, während der erfahrene Sachverständige mir zugeben wird, dass die Anzahl der chronischen operirten Fälle im Verhältniss zu den acuten eine keineswegs grosse ist.

Es ist selbstverständlich, dass nur ein Bruchtheil aller operirten chronischen Fälle die sogenannte "Radicaloperation" betrifft. Um nun eine genaue Würdigung der Anzabl der "Radicaloperationen" in den chronischen Fällen zu erhalten, habe ich

1) Die Anmerkungen zur vorliegenden wortlichen Wiedergabe meines Vortrages sind erst später hinzugefugt. 
das procentualische Verhältniss aller Operationen zu der Summe der Ohreiterungen überhaupt berechnet and hierzu die letzten 4 Jahre ausgewählt, wozu ich bemerke, dass hierbei die in Preussen iblichen sogenannten Etatsjahre von April bis April gemeint sind.

Es geschah dies hauptsächlich darum, weil in diese Periode die seit dem Jahre 1895 von mir angewandte Behandlung mit Ausspritzungen von Formalinlösung fällt. Diese Behandlung hatte einen doppelten Vortheil, indem es mir einerseits gelang, eine grössere Zahl chronischer Falle auch ohne Operation zur Heilung zu bringen, anderseits da, wo das Mittel einen negativen Erfolg hatte, die Indication zur Operation genaner zu präoisiren.

Es ergaben sich in der angegebenen Zeit nun folgende Zahlenverhältnisse:

1895/96: Acute Fälle 648 mit 86 Operationen $=11,72$ Proc. Chron. $=1413=118=2,35=$

Summa 2061

1896/97: Acute Fälle 528 mit 66 Operationen $=12,5=$ Chron. $=1208=85==7,03=$

Summa 1736

1897/98: Acute Fälle 581 mit 69 Operationen $=11,87=$

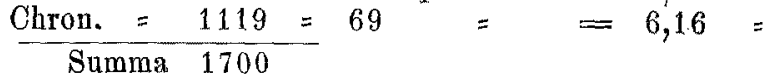

1898/99: Acute Fälle 530 mit 61 Operationen $=11,51=$ Chron: $=1131=90==7,95=$

Ohne an dieser Stelle auf die jährliche Abnahme der Fälle von Mittelohreiterungen näher einzugehen, will ich nur erwähnen, dass gegenwärtig vier. Polikliniken für Obrenkranke in nächster Nähe der Universitäts-Ohrenklinik bestehen. Diese Thatsache kann jedoch kaum allein die mit dem Jahre 1896/97 eintretende plötzliche Abnahme und die allmähliche weitere Verminderung: der Ohreiterungen erklären, da die Zahl sämmtlicher neuer Kranken keineswegs abgenommen hat, sondern in der Zeit von 1895-99 eine Zunahme von 6536 anf 6704 ergiebt.

Die Zahlen der Procente sprechen wohl fur sich selbst laut genug und zeigen, dass die Zahl der Operationen in den chronischen eine weit geringere ist, als die in den acuten Fallen: im Jahre 1897/98 beträgt dieselbe beinahe die Hälfte der in den 
acuten Fällen Operirten. Es ist ferner aus den Zahlen zu ersehen, wie klein der absolute Procentsatz der Operationen in den chronischen Fällen überhaupt ist, und dass auch die acuten Fälle verhältnissmässig wenig Operationen erforderten.

Ein statistischer Vergleich dieser vierjährigen Formalinperiode mit den früheren Jahrgängen würde keine sicheren Resultate ergeben, weil die meisten chronischen Eiterungen in der Poliklinik (ont-patients) und nur wenige auf der stationären Klinik mit Formalinlösung behandelt wurden und daher, wie es leider in jeder Poliklinik geschieht, nicht regelmässig wiederkehrten. Wir hatten jedoch den allgemeinen Lindruck, dass der Erfolg der Formalinbehandlung ein grösserer war als die mit anderen Mitteln, namentlich mit Borsäurelösungen erzielten Resultate.

Ganz besonders hat sich diese Behandlung bewährt in den ohne jede drohenden Symptome verlaufenden "kalten" Fällen, wo nur wegen der dauernd fötiden Beschaffenheit des Secretes der Verdacht auf ein tieferes Ohrenleiden vorlag. Die allgemeine Regel war, dass da, wo die dureh $4-6$ Wochen ${ }^{1)}$ vorgenommene sorgfältige tägliche Behandlung absolut keine Veränderung des Foetors bewirkte, die Operation stets mehr oder weniger schwere Erkrankungen des Felsenbeins zeigte (Empyem mit Granulationen, Caries, resp. Nekrose, Cholesteatom), abgesehen von den bekannten, so häufig und unerwartet sich einfindenden Erkrankungen in den benachbarten Räumen der Schädelhöhle. - Das Formalin hat den doppelten Vortheil, dass es nicht nur ansserordentlich wirksam, sondern auoh sehr billig ist.

Die Stärke der zn den Einspritzungen ${ }^{2}$ ) von mir benützten Lösung ist 15-20 Tropfen auf 1 Liter abgekochten Wassers. Besondere und andauernde Reizerscheinungen haben wir dabei niemals beobachtet; das einzig Unangenehme, namentlich bei Kindern, ist, dass das Mittel durch Abfliessen dureh die Tuba E.

1) Es ist hierbei zu bemerken, dass es sich meist um Falle handelte, wo schon lange Zeit vorher die verschiedensten Mittel von anderen Aerzten angewendet waren. Auch muss bervorgehoben werden, dass in der bei weitem grösseren Zahl der Falle die Formalinbehandlung schon darch den Widerstand der Patienten gegen die Operation auf Monate ausgedehnt warde.

2) Ich erlaube mir hier noch einmal anf die von mir (Zur conservativen und operativen Behandlung der chronischen Mittelohreiterungen. Therapeut. Monatsschr. Aug. 1897) zur Vermeidung von Schwindel, resp. Verletzungen an der Labyrinthwand empfohlene Gummicanäle aufmerksam zu machen, welche am Ohrende geschlossen, nur Seitenlöcher besitzt und au jeder Ohrenspritze leicht anzubringen ist. 
188 XVII. LUCAE, Stat. Beitr. zur oper. Eröffnung des Proc. mast. u. s.w.

mitunter vorübergehende Sohmerzen im Pharynx hervorbringt, was sich jedoeh durch Gurgeln mit Wasser leicht beseitigen lässt. Eventuell kann bei der so grossen antiseptischen Kraft des Formalins eine noch sehwäehere Lösung benützt werden. ${ }^{1}$ )

Mr President and Gentlemen, As $J$ think in German $J$ have spoken in my native tongue. But $I$ now only wish to say some words for the British ears not understanding German.

I am of the opinion that the opening of the mastoid resp. of the whole middle ear is a very important help in the treatment of Chronic suppurative Otitis media. But one may also get on in plenty of eases by non operating treatment. I beg to add that instead of being proud of saying: "J have operated on so many patients", one should be prouder of saying: "J have cured so many patients also without operating."

1) Auch zur Nachbehandlung hat mir die Einführung von Gazetampons, angefeuchtet mit derselben Lösung, in gewissen Făllen sehr gute Dienste geleistet. 